\title{
New operational taxonomic units of Enterocytozoon in three marsupial species
}

\author{
Yan Zhang ${ }^{1}$, Anson V. Koehler ${ }^{1 *}$ (D), Tao Wang ${ }^{1}$, Shane R. Haydon ${ }^{2}$ and Robin B. Gasser ${ }^{1 *}$
}

\begin{abstract}
Background: Enterocytozoon bieneusi is a microsporidian, commonly found in animals, including humans, in various countries. However, there is scant information about this microorganism in Australasia. In the present study, we conducted the first molecular epidemiological investigation of E. bieneusi in three species of marsupials (Macropus giganteus, Vombatus ursinus and Wallabia bicolor) living in the catchment regions which supply the city of Melbourne with drinking water.

Methods: Genomic DNAs were extracted from 1365 individual faecal deposits from these marsupials, including common wombat $(n=315)$, eastern grey kangaroo $(n=647)$ and swamp wallaby $(n=403)$ from 11 catchment areas, and then individually tested using a nested PCR-based sequencing approach employing the internal transcribed spacer (ITS) and small subunit (SSU) of nuclear ribosomal DNA as genetic markers.

Results: Enterocytozoon bieneusi was detected in 19 of the 1365 faecal samples (1.39\%) from wombat $(n=1)$, kangaroos $(n=13)$ and wallabies $(n=5)$. The analysis of ITS sequence data revealed a known (designated NCF2) and four new (MWC_m1 to MWC_m4) genotypes of E. bieneusi. Phylogenetic analysis of ITS sequence data sets showed that MWC_m1 (from wombat) clustered with NCF2, whereas genotypes MWC_m2 (kangaroo and wallaby), MWC_m3 (wallaby) and MWC_m4 (kangaroo) formed a new, divergent clade. Phylogenetic analysis of SSU sequence data revealed that genotypes MWC_m3 and MWC_m4 formed a clade that was distinct from E. bieneusi. The genetic distinctiveness of these two genotypes suggests that they represent a new species of Enterocytozoon.

Conclusions: Further investigations of Enterocytozoon spp. from macropods and other animals will assist in clarifying the taxonomy and epidemiology of these species in Australia and elsewhere, and in assessing the public health risk of enterocytozoonosis.
\end{abstract}

Keywords: Enterocytozoon bieneusi, Operational taxonomic units, Genotypes, Prevalence, Eastern grey kangaroo, Swamp wallaby, Common wombat, Australia

\section{Background}

Enterocytozoon bieneusi (Microsporidia) is a tiny $(0.5 \times 1.5$ $\mu \mathrm{m})$ [1] fungus or fungus-like microorganism [2, 3]. This microorganism infects the gastrointestinal tracts of mammals, and can cause acute or chronic diarrhoea, wasting and/or malabsorption [4-7]. However, some individuals infected with E. bieneusi do not exhibit clinical signs, and can thus represent carriers with the potential of disseminating E. bieneusi spores (via faeces) into the environment and/or to other people or animals [8]. As an opportunistic pathogen, E. bieneusi infects both immunocompromised

\footnotetext{
* Correspondence: anson.koehler@unimelb.edu.au; robinbg@unimelb.edu.au 'Department of Veterinary Biosciences, Melbourne Veterinary School, Faculty of Veterinary and Agricultural Sciences, The University of Melbourne, Parkville, Victoria 3010, Australia

Full list of author information is available at the end of the article
}

(e.g. HIV/AIDS and organ transplant patients) and immunocompetent people [1], and has been recorded in at least 74 species of animals (e.g. artiodactyls, carnivores, lagomorphs, perissodactyls, primates and rodents) [9].

The accurate classification of E. bieneusi from biological matrices (faeces, soil and water) is not possible using phenetic (e.g. morphological) approaches, such that molecular tools need to be employed. Specifically, PCR-based sequencing of the internal transcribed spacer (ITS) of nuclear ribosomal DNA is an established, widely used method for the specific and genotypic identification and taxonomic classification of E. bieneusi [1]. Using this tool, more than 200 genotypes of $E$. bieneusi have been defined, 52 of which have been recorded only in humans, 34 in both humans and animals, 103 exclusively 
in animals (including artiodactyls, carnivores, lagomorphs, perissodactyls, primates and rodents) and 36 in water samples [9]. To date, there are only two studies reporting E. bieneusi in marsupials, but both studies involved captive kangaroos in China [10,11].

Some genotypes, including D, EbpA, J, K and Type IV, have been identified in both humans and animals (e.g. bovids, canids and primates), and are thus recognised as potentially zoonotic $[1,12]$. However, there has been no experimentation to demonstrate cross-species transmissibility [13]. Nonetheless, particular attention needs to be paid to these 'zoonotic' genotypes in the context of foodor water-borne disease (enterocytozoonosis) [14, 15]. Indeed, E. bieneusi is recognised as a Category B Priority Pathogen by National Institute of Allergy and Infectious Diseases (NIAID) and a 'water contaminant candidate' by the United States Environmental Protection Agency [6]. However, E. bieneusi is not mentioned in the Australian Drinking Water Guidelines (www.nhmrc.gov.au/guidelines-publications/eh52; accessed 28 February 2018), and there is no detailed information on its relevance as a waterborne pathogen for most countries.

Melbourne (population: $\sim 4$ million people) in the state of Victoria, Australia, is one of the few cities in the world that receives largely unfiltered drinking water from protected wilderness catchment areas. The management of these areas includes long water retention times, a monitoring programme for pathogens in source water and restricted access for humans. These catchment areas contain native and feral animals, and the monitoring of zoonotic microbes is central to the prevention of waterborne disease or disease outbreaks.

Since 1997, we have been investigating selected waterborne microbes, including Cryptosporidium and Giardia, in faecal samples from animals inhabiting these catchment areas [16]. As there was no published information on $E$. bieneusi in Australia, except for case studies of human enterocytozoonosis in HIV/AIDS patients (e.g. [17, 18]), we extended our monitoring programme to include this microbe. Recently, we conducted the first molecular epidemiological survey of $E$. bieneusi in wild fallow, red and sambar deer [19], which are abundant animals within Melbourne's drinking water catchment areas (cf. $[16,20])$. Enterocytozoon bieneusi was detected in 25 of the 610 (4.1\%) samples tested, and was found exclusively in sambar deer. Other animals, such as marsupials, rabbits, canids and birds, are also relatively pronounced in these catchments, and might represent potential carriers of E. bieneusi. For this reason, in this study, we explored the prevalence, genetic diversity and zoonotic potential of Enterocytozoon in wombats, kangaroos and wallabies using a nested PCR-based sequencing of ITS and small subunit of nuclear ribosomal DNA gene (SSL), and phylogenetic analysis of sequence data sets. We discuss the research findings in the context of Enterocytozoon taxonomy and epidemiology.

\section{Methods \\ Melbourne's water catchments}

Natural water catchments, covering more than 160,000 hectares, supply drinking water to $\sim 4$ million people in the city of Melbourne and environs. Humans and domestic animals have restricted access to most catchment areas. Water is treated in accord with national and international guidelines (Australian Drinking Water Guidelines www.nhmrc.gov.au/guidelines-publications/eh52; accessed February 2018). The catchment areas studied here were Armstrong (AM) $37^{\circ} 38^{\prime} \mathrm{S}, 145^{\circ} 51^{\prime} \mathrm{E}$; Cardinia (CA) $37^{\circ} 47^{\prime} \mathrm{S}, 145^{\circ} 24^{\prime} \mathrm{E}$; Greenvale (GV) $37^{\circ} 37^{\prime} \mathrm{S}, 144^{\circ} 54^{\prime} \mathrm{E}$; Maroondah (MR) $37^{\circ} 38^{\prime} \mathrm{S}, 145^{\circ} 33^{\prime} \mathrm{E}$; O'Shannassy (OS) $37^{\circ} 40^{\prime} \mathrm{S}, 145^{\circ} 48^{\prime} \mathrm{E}$; Silvan (SV) $37^{\circ} 50^{\prime} \mathrm{S}, 145^{\circ} 25^{\prime} \mathrm{E}$; Sugarloaf (SL) $37^{\circ} 40^{\prime} \mathrm{S}, 145^{\circ} 18^{\prime} \mathrm{E}$; Tarago (TAR) $37^{\circ} 59^{\prime} \mathrm{S}, 145^{\circ} 55^{\prime} \mathrm{E}$; Thompson (TH) $37^{\circ} 47^{\prime} \mathrm{S}, 146^{\circ} 21^{\prime} \mathrm{E}$; Upper Yarra (UY) $37^{\circ} 40^{\prime} \mathrm{S}, 145^{\circ} 55^{\prime} \mathrm{E}$ and Yan Yean (YY) $37^{\circ} 33^{\prime} \mathrm{S}, 145^{\circ} 08^{\prime} \mathrm{E}$ (cf. [16]. CA, GV and SV act as storage facilities for the larger catchments; MR, OS, TH and UY are located in the densely forested Yarra Ranges catchment; TAR is an 'open' water-supply catchment abutting farmland; and $\mathrm{YY}$ is a much smaller catchment surrounded by residential areas.

\section{Samples and DNA isolation}

A total of 1365 faecal deposits from marsupials, including common wombat (Vombatus ursinus; $n=315$ ), eastern grey kangaroo (Macropus giganteus; $n=647$ ) and swamp wallaby (Wallabia bicolor; $n=403$ ) were collected from eleven water catchments from September 2009 to March 2017 (Additional file 1: Table S1). Genomic DNA was isolated directly from $0.25 \mathrm{~g}$ of each of the 1365 samples using the PowerSoil kit (MoBio, Carlsbad, CA, USA) according to the manufacturer's instructions. The host origin of scats was identified using a field guide [21], and verified by testing faecal DNA employing a nested PCR-based sequencing approach using the mitochondrial cytochrome $b$ gene (cf. [22]).

\section{Nested PCR-based sequencing of E. bieneusi ITS}

From individual faecal DNA samples, the internal transcribed spacer (ITS) of nuclear ribosomal DNA of $E$. bieneusi was specifically amplified using degenerate primers originally published by Katzwinkel-Wladarsch et al. [23]. In the first round, primers MSP-1 (forward: 5'-TGA ATG KGT CCC TGT-3') and MSP-2B (reverse: 5'-GTT CAT TCG CAC TAC T-3') were employed to amplify 601 bp of ITS plus flanking gene sequences. In the second round, primers MSP-3 (forward: 5'-GGA ATT CAC ACC GCC CGT CRY TAT-3') and MSP-4B (reverse: 5'-CCA AGC TTA TGC TTA AGT CCA GGG 
AG-3') were used to amplify a product of $\sim 535$ bp containing $130 \mathrm{bp}$ of the 3 '-end of the small subunit ( $S S U$ ) of the nuclear rDNA gene, $\sim 243 \mathrm{bp}$ of the internal transcribed spacer (ITS) plus $162 \mathrm{bp}$ of the 5 -region of the large subunit $(L S U)$ rDNA gene.

Nested PCR for amplification of ITS was conducted in $50 \mu \mathrm{l}$ in a standard buffer containing $2.0 \mu \mathrm{M} \mathrm{MgCl}, 0.4$ mM dNTPs, 50 pmol of each primer, 1.25 U of MangoTaq polymerase (Bioline, London, UK) and DNA template - except for the negative (no-template) controls. Known test-positive, test-negative and no-template controls were included in every round of every PCR run. The cycling conditions for both primary and secondary (nested) PCRs were: $94{ }^{\circ} \mathrm{C}$ for $5 \mathrm{~min}$ (initial denaturation), followed by 35 cycles of $94{ }^{\circ} \mathrm{C}$ for $45 \mathrm{~s}$ (denaturation), $54{ }^{\circ} \mathrm{C}$ for $45 \mathrm{~s}$ (annealing) and $72{ }^{\circ} \mathrm{C}$ for $1 \mathrm{~min}$ (extension), followed by $72{ }^{\circ} \mathrm{C}$ for $10 \mathrm{~min}$ (final extension).

Amplicons were examined on ethidium bromide-stained $1.5 \%$ agarose gels using TBE $(65 \mathrm{mM}$ Tris- $\mathrm{HCl}, 27 \mathrm{mM}$ boric acid, 1 mM EDTA, pH 9; Bio-Rad, Hercules, CA, USA) as the buffer, and their size estimated using a 100 bp-DNA ladder (Promega, Madison, WI, USA) as a reference. Amplicons were individually treated with ExoSAP-IT (Affymetrix, Santa Clara, CA, USA) according to the manufacturer's instructions and directly sequenced (BigDye Terminator v.3.1 chemistry, Applied Biosystems, Foster City, CA, USA) using primers MSP-3 and MSP-4B in separate reactions. ITS sequences were aligned and examined using the program Geneious v.10 [24], and compared with other sequences acquired from the GenBank database (Additional file 2: Table S2). Sequence types or genotypes of $E$. bieneusi were named according to recent recommendations $[1,12]$. ITS sequences were deposited in the GenBank database (NCBI) (accession nos. MG976812-MG976817).

\section{Nested PCR-based sequencing of E. bieneusi SSU}

Selected genomic DNA samples that were test-positive for ITS were subjected to nested PCR-based sequencing of the small subunit of nuclear ribosomal DNA gene $(S S U)$. In the first round of PCR, primers $\mathrm{C} 1$ (forward: 5'-CAC CAG GTT GAT TCT GCC-3') [25] and EBssuR2 (reverse: 5'-AAG CTC TTC ATC CCT ATG ACC ATC-3') (designed in the present study) were used to amplify 1216 bp of SSU. In the second round, primers EBIEF1 (forward: 5'-GAA ACT TGT CCA CTC CTT ACG-3') and EBIER1, 5'-CCA TGC ACC ACT CCT GCC ATT-3') [26] were employed to amplify a product of $\sim 610 \mathrm{bp}$ of $S S U$. PCR was conducted in $50 \mu \mathrm{l}$ using the same buffer as employed for ITS. Known test-positive, test-negative and no-template controls were included in every round of every PCR run. For the first round, cycling conditions were: $94{ }^{\circ} \mathrm{C}$ for $5 \mathrm{~min}$ (initial denaturation), followed by 35 cycles of $94{ }^{\circ} \mathrm{C}$ for $1 \mathrm{~min}$ (denaturation), 56
${ }^{\circ} \mathrm{C}$ for $1 \mathrm{~min}$ (annealing) and $72{ }^{\circ} \mathrm{C}$ for 1 min (extension), followed by $72{ }^{\circ} \mathrm{C}$ for $10 \mathrm{~min}$ (final extension). For the second round, cycling conditions were same except for denaturation $\left(94{ }^{\circ} \mathrm{C}\right.$ for $\left.45 \mathrm{~s}\right)$ and annealing $\left(64{ }^{\circ} \mathrm{C}\right.$ for $\left.45 \mathrm{~s}\right)$. Amplicons were examined, sized, purified and sequenced (same methods as for ITS) using primers EBIEF1 and EBIER1 in separate reactions. SSU sequences were examined and aligned using Geneious v.10 [24] and then compared with other sequences from the GenBank database (Additional file 3: Table S3). SSU sequences were deposited in the GenBank database (NCBI) (accession nos. MG976584-MG976586).

\section{Phylogenetic analysis of sequence data}

ITS and SSU sequences from this and previous studies (cf. Additional file 2: Table S2 and Additional file 3: Table S3) were aligned separately over consensus lengths of 280 and 609 positions, respectively, and then subjected to phylogenetic analyses using the Bayesian inference (BI) and Monte Carlo Markov Chain (MCMC) methods in MrBayes v.3.2.3 [27]. The Akaike Information Criteria (AIC) test in jModeltest v.2.1.7 [28] was used to evaluate the likelihood parameters set for BI analysis. Posterior probability (pp) values were calculated by running 2,000,000 generations with four simultaneous tree-building chains, with trees saved every one hundredth generation. A 50\% majority rule consensus tree for each analysis was constructed based on the final $75 \%$ of trees generated by BI. Enterocytozoon bieneusi clades and subclades were assigned using an established classification system $[29,30]$.

\section{Results}

Enterocytozoon DNA was detected in 19 of 1365 (1.4\%) faecal samples from wild marsupials, including eastern grey kangaroo $(n=13)$, swamp wallaby $(n=5)$ and common wombat $(n=1)$ from five of 11 water catchment areas by nested PCR of ITS (Additional file 1: Table S1). For all 11 catchments, the prevalences of Enterocytozoon were $2.0 \%(13 / 647)$ in the eastern grey kangaroo, $1.2 \%$ $(5 / 403)$ in the swamp wallaby and $0.3 \%(1 / 315)$ in the common wombat. Within individual catchments, the highest prevalence $(4.2 \% ; 1 / 24)$ was recorded in catchment TAR, compared with $1.3-4.0 \%$ in the four other catchments (GV, MR, SV and YY). Test-positive samples were found in all seasons, with the highest prevalence $(2.5 \% ; 7 / 282)$ of Enterocytozoon in Spring; prevalences varied from $0-3.7 \%$ among years.

The sequencing of the 19 ITS amplicons (240-245 bp) and comparisons with reference sequences in the GenBank database identified five distinct sequence types (genotypes). One $E$. bieneusi genotype from the eastern grey kangaroo was NCF2 (matching a known sequence with accession no. KT750162) [31], and four were new genotypes (designated 
MWC_m1 to MWC_m4). Genotype MWC_m1 was from a common wombat; genotypes MWC_m2 and MWC_m3 were from swamp wallabies; and genotypes MWC_m2 and MWC_m4 were from eastern grey kangaroos. The mean G $+C$ content (11.0\%) of ITS sequences representing genotypes MWC_m2 to MWC_m4 was substantially less than those of the nine established Groups (1 to 9) of E. bieneusi genotypes (39.0-55.6\%).

Genotype MWC_m1 from wombat differed by $1 \mathrm{bp}$ (242/243; 99.6\%) from the sequence with accession no. KT750162 (E. bieneusi genotype NCF2 from arctic fox) [31]. Genotypes MWC_m2, MWC_m3 and MWC_m4 each differed by 15 bp $(229 / 244 ; 94.2 \%), 13$ bp $(232 / 245$; 94.7\%) and 2 bp $(243 / 245 ; 99.2 \%)$ from the sequence with accession number KY706128 (i.e., representing $E$. bieneusi genotype CSK2 from red kangaroo (Macropus rufus); [11]). MWC_m4 was a "dominant" genotype in all three marsupial species studied here, having been detected in 6,2 and 3 samples from catchment areas GV, $\mathrm{SV}$ and YY, respectively (Table 1 ).

The ITS sequence data representing the five distinct genotypes (representing all 19 samples) were subjected to a phylogenetic analysis which included representative sequence data for 9 established groups of E. bieneusi (Fig. 1). The sequences of genotypes defined in this study were related to the currently recognised Groups (1 to 9) of E. bieneusi. Genotypes NCF2 and MWC_m1 could be assigned to Group 1, with strong statistical support ( $\mathrm{pp}=0.98)$. Genotypes CHK1 and CHK2 from an indeterminate species of macropod [10] as well as CSK1 from red kangaroo [11] in China all formed a new clade, namely Group 10, with strong statistical support (pp = 1.00). Genotypes MWC_m2 to MWC_m4 formed a new clade, namely Group $11(\mathrm{pp}=1.00)$. Although Groups 1 , 3,5 to 11 were strongly supported ( $\mathrm{pp}=0.97$ to 1.00 ), Groups 2 and 4 were not ( $\mathrm{pp}=0.55$ to 0.90 ).

The relationship between novel genotypes MWC_m2 to MWC_m4 in this study and other species representing the family Enterocytozoonidae [32, 33] was inferred from a separate phylogenetic analysis of SSU sequence data using BI (Fig. 2; pairwise comparison of nucleotide differences given in Additional file 4: Table S4). The major clades were well-supported ( $\mathrm{pp}=0.97$ to 1.00 ), most genotypes (MWC_m1, MWC_m3, MWC_m4 and NCF2; cf. Fig. 1) identified in this study represented the E. bieneusi clade. However, within this clade, there was limited support amongst most taxa. Representatives of genotypes MWC_m3 and MWC_4 clustered together, and formed a separate clade $(\mathrm{pp}=1.00)$ to the exclusion of all taxa representing E. bieneusi (Fig. 2).

Table 1 Genotypic designations for the operational taxonomic units (OTUs) of Enterocytozoon characterised by their internal transcribed spacer (ITS) of nuclear ribosomal DNA sequences (accession nos. listed) from 19 of 1365 individual faecal deposits (sample codes listed) from common wombats, eastern grey kangaroos and swamp wallabies, collected from five of Melbourne's water catchment areas in different seasons and years

\begin{tabular}{|c|c|c|c|c|c|c|}
\hline OTU/genotypic designation $^{a}$ & GenBank ID & Sample code & Host & Catchment area & Season & Year \\
\hline NCF2 & MG976814 & GV6110 & Kangaroo & Greenvale & Winter & 2015 \\
\hline MWC_m1 & MG976816 & SV5961 & Wombat & Silvan & Autumn & 2015 \\
\hline MWC_m2 & MG976812 & SV4324 & Kangaroo & Silvan & Summer & 2014 \\
\hline MWC_m2 & MG976815 & MR3417 & Wallaby & Maroondah & Autumn & 2013 \\
\hline MWC_m2 & MG976815 & MR3433 & Wallaby & Maroondah & Autumn & 2013 \\
\hline MWC_m2 & MG976815 & TAR2172 & Wallaby & Tarago & Winter & 2011 \\
\hline MWC_m3 & MG976817 & MR7053 & Wallaby & Maroondah & Winter & 2016 \\
\hline MWC_m3 & MG976817 & MR7114 & Wallaby & Maroondah & Winter & 2016 \\
\hline MWC_m4 & MG976813 & GV4446 & Kangaroo & Greenvale & Autumn & 2014 \\
\hline MWC_m4 & MG976813 & GV4494 & Kangaroo & Greenvale & Autumn & 2014 \\
\hline MWC_m4 & MG976813 & GV4998 & Kangaroo & Greenvale & Spring & 2014 \\
\hline MWC_m4 & MG976813 & GV5007 & Kangaroo & Greenvale & Spring & 2014 \\
\hline MWC_m4 & MG976813 & GV5050 & Kangaroo & Greenvale & Spring & 2014 \\
\hline MWC_m4 & MG976813 & GV3007 & Kangaroo & Greenvale & Spring & 2012 \\
\hline MWC_m4 & MG976813 & SV3758 & Kangaroo & Silvan & Winter & 2013 \\
\hline MWC_m4 & MG976813 & SV4932 & Kangaroo & Silvan & Winter & 2014 \\
\hline MWC_m4 & MG976813 & YY3092 & Kangaroo & Yan Yean & Spring & 2012 \\
\hline MWC_m4 & MG976813 & YY3095 & Kangaroo & Yan Yean & Spring & 2012 \\
\hline MWC_m4 & MG976813 & YY3117 & Kangaroo & Yan Yean & Spring & 2012 \\
\hline
\end{tabular}

${ }^{a}$ Genotypes MWC_m1 to MWC_m4 are all novel 


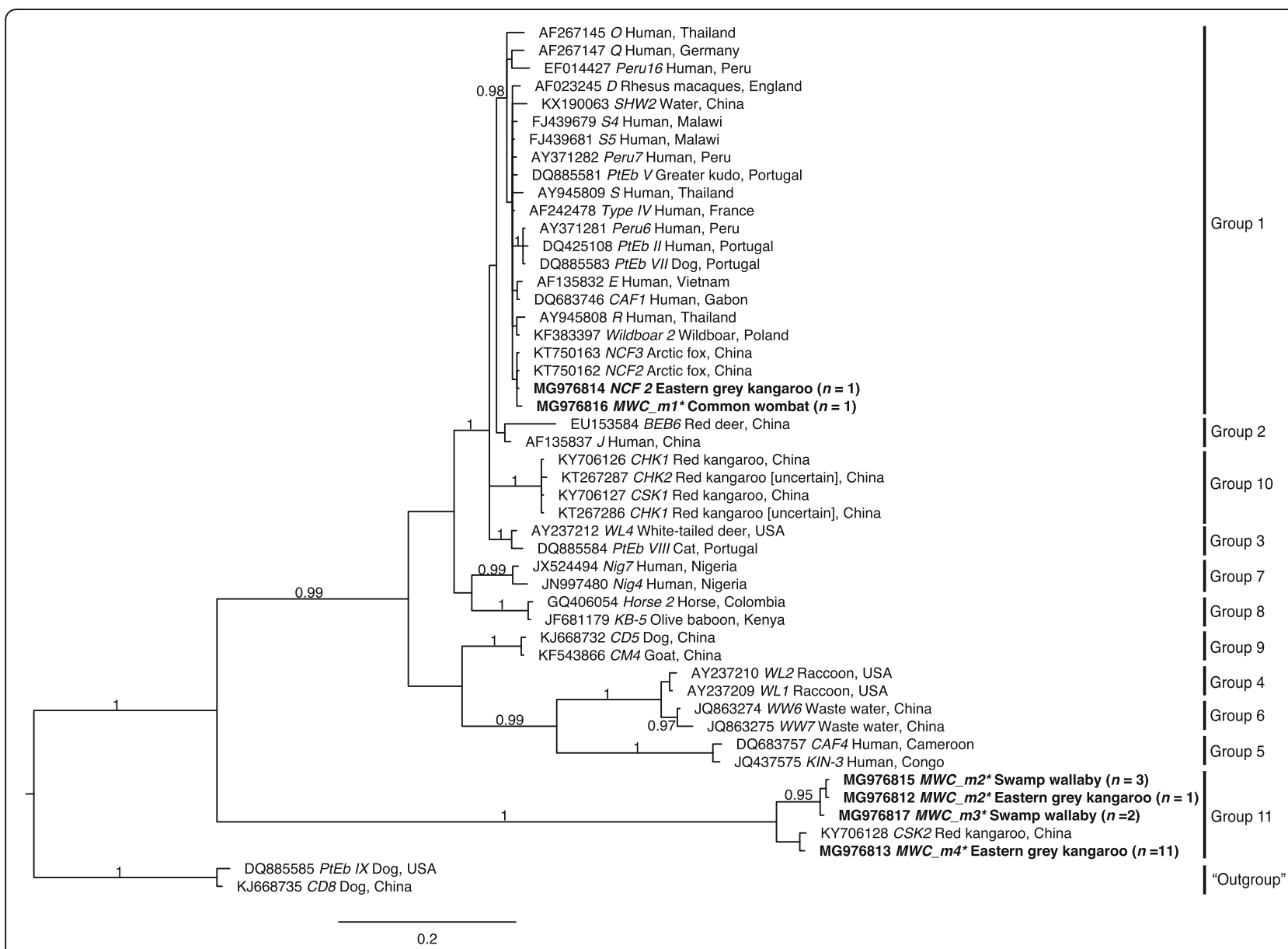

Fig. 1 Phylogenetic analysis of internal transcribed spacer (ITS) of nuclear ribosomal DNA sequence data (Additional file 2: Table S2) by Bayesian inference (BI). Included here are ITS sequences of (i) E. bieneusi genotypes representing all currently recognised Groups (1 to 9) from the published literature; (ii) five genotypes of Enterocytozoon identified in the present study (bold-type); and (iii) the outgroup taxa. Groups 10 and 11 are proposed based on the results of the present analysis. Statistically significant posterior probabilities (pp) are indicated on branches. The scale-bar represents the number of substitutions per site

\section{Discussion}

This is the first molecular investigation of Enterocytozoon of marsupials in Australia. PCR-based sequencing of all 19 amplicons from 1365 faecal DNA samples (prevalence: $1.39 \%$ ) revealed five operational taxonomic units (OTUs) of Enterocytozoon from a common wombat $(n=1)$, swamp wallabies $(n=5)$ and eastern grey kangaroos $(n=13)$ in Australia. In this study, we used oligonucleotide primers designed to flanking $S S U$ and $L S U$ regions of $E$. bieneusi to PCR-amplify rDNA products of 535-537 bp [23]. ITS sequence lengths obtained here were consistent with those (240-245 bp) for all nine recognised groups of $E$. bieneusi [29, 30], but distinct from other microsporidians, including Encephalitozoon spp. (E. cuniculi, E. hellem and E. intestinalis; 28-46 bp) [34], Nucleospora cyclopteri (261 bp) [35], Nucleospora salmonis (261 bp) [36] and Obruspora papernae (227 bp) [37].

When the 19 ITS amplicons derived from faecal DNAs from these marsupials were sequenced, and the five distinct sequences obtained were compared with those of $E$. bieneusi genotypes $(n=43)$ representing all nine recognised groups $[29,30]$, we identified genotype NCF2 from an eastern grey kangaroo and MWC_m1 from a common wombat. Phylogenetic analysis of ITS data showed that these two OTUs both clustered with genotypes recorded previously in humans, arctic fox, dog and wild boar [9] (Group 1; see Fig. 1). The relatedness of these OTUs with genotypes from humans (CAF1, D, E, Peru6, PtEb II, Q, S4, S5 and Type IV) might be an indication that they have zoonotic potential, but further study of many more samples from animals and humans is required to assess this proposal.

By contrast, the ITS sequences derived from the other three OTUs (MWC_m2 to MWC_m4 from eastern grey kangaroos or swamp wallabies) were highly divergent (57.4-64.4\%) from those of any member of any of the ten recognised genotypic groups of E. bieneusi (Fig. 1). Indeed, this divergence as well as AT-richness (89\%) in 


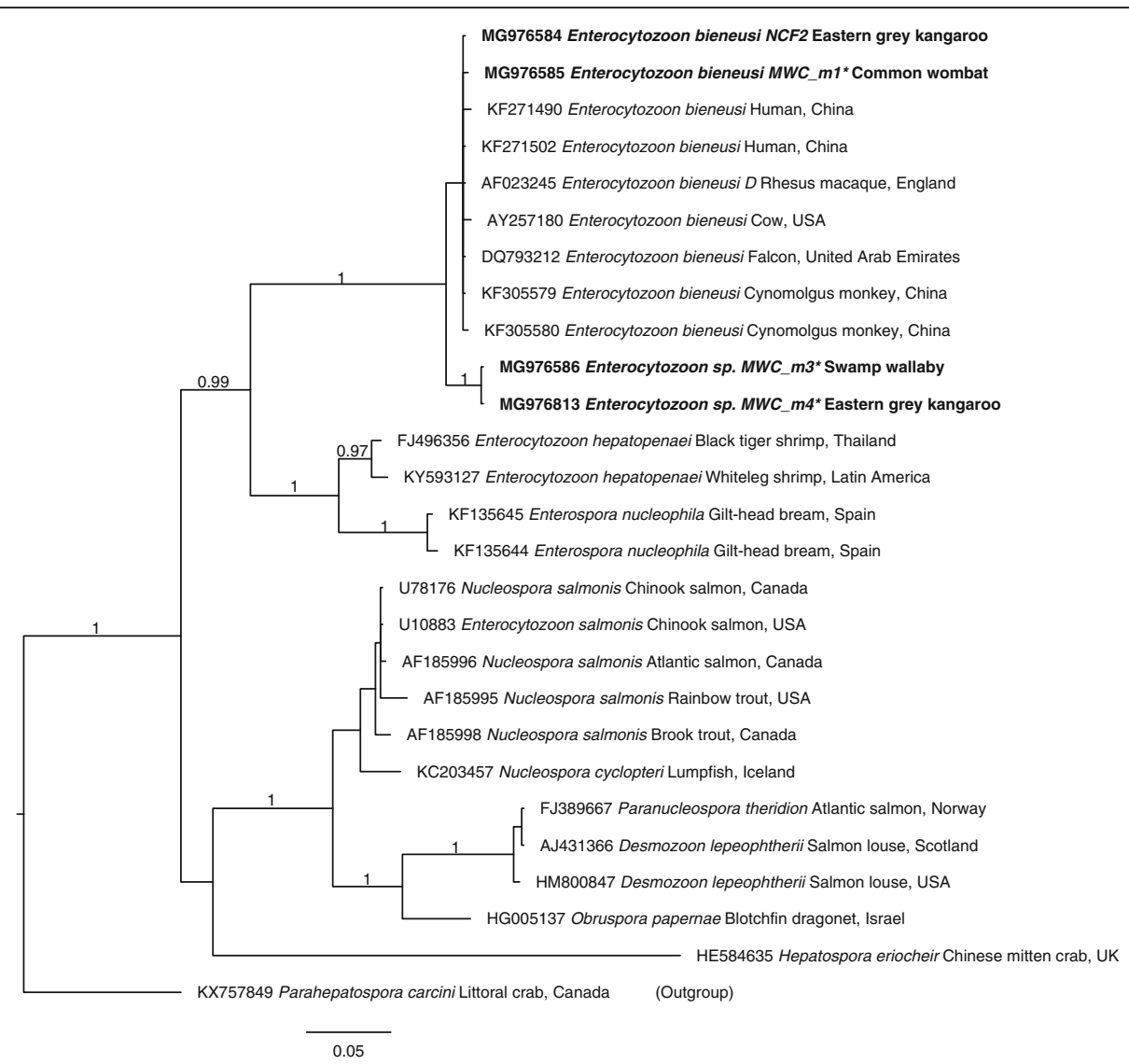

Fig. 2 Phylogenetic analysis of small subunit of rDNA sequences (SSU) DNA sequence data (Additional file 3: Table S3) by Bayesian inference (BI). Included here are SSU sequences of (i) key taxa of the family Enterocytozoonidae, including representatives of E. bieneusi from the published literature; (ii) four operational taxonomic units (OTUs) or genotypes of Enterocytozoon identified in the present study (bold-type; cf. Fig. 1); and (iii) the outgroup: Parahepatospora carcini. Statistically significant posterior probabilities (pp) are indicated on branches. The scale-bar represents the number of substitutions per site

ITS (but not in flanking SSU or LSU gene regions) did not allow a reliable alignment of homologous characters in the overall sequence alignment employed for phylogenetic analysis (Fig. 1), preventing a sound inference regarding the species or genotypic status of these three OTUs, particularly given their position relative to the outgroups (cf. Fig. 1).

Clearly, the sequences of the primers used in PCR were consistent with $E$. bieneusi to allow specific amplification, but sequence divergence in ITS prevented us from being able to reliably assign these three OTUs to any of the ten genotypic groups of E. bieneusi. Thus, we provisionally assigned them to a new group (Group 11) (Fig. 1). Given the substantial sequence divergence in ITS, we elected to use a more conserved rDNA gene region to investigate the taxonomic status of these four OTUs further. To do this, we designed new (forward and reverse) primers to SSU to PCR-amplify products of $\sim 610$ bp from faecal DNA samples in which we had identified representative, novel OTUs (MWC_m1, MWC_m3 and MWC_m4). Following the sequencing of these amplicons, we obtained SSU sequences which could be aligned (over a consensus length of 609 positions) with publicly available sequences $(n=23 ; 6$ February 2018) representing seven genera (Enterozytozoon, Enterospora, Hepatospora, Nucleospora, Obruspora, Parahepatospora and Paranucleospora) and eight recognised species (including E. bieneusi, E. hepatopenaei, E. salmonis (syn. Nucleospora salmonis; [36]), Enterospora nucleophila, Hepatospora eriocheir, Nucleospora cyclopteri, Obruspora papernae and Paranucleospora theridion (syn. Desmozoon lepeophtherii; cf. [32]) and an outgroup, Parahepatospora carcini, within the family Enterocytozoonidae (Microsporidia) (Additional file 3: Table S3). In this case, homologous characters could be reliably aligned, and the phylogenetic analysis of the SSU data set showed that the novel OTUs (genotypes MWC_m3 and MWC_m4) grouped, with strong nodal support ( $\mathrm{pp}=1.00)$, with members of the genus Enterocytozoon, to the exclusion of all 16 other enterocytozoonid taxa included (Fig. 2). This analysis showed clearly that genotype MWC_m1 clustered with other members of the E. bieneusi-clade and thus 
represented E. bieneusi, and that MWC_m3 \& MWC_m4 represented a new and distinct clade. As sequence variation $(0-0.6 \%)$ representing the genetic sub-structuring within $E$. bieneusi was substantially less than differences (2.8-3.2\%) between E. bieneusi genotypes and MWC_m3 \& MWC_m4 upon pairwise comparison (Fig. 2), we propose that the latter two genotypes represent a novel species of Enterocytozoon. However, further work is required to sequence SSU from many more representatives of Enterocytozoon, in order to conduct a comprehensive phylogenetic analysis. The naming of a new species would need to follow all of the criteria set by the International Code of Nomenclature for Algae, Fungi and Plants [38].

There has been ongoing discussion about the species status of members of the genus Enterocytozoon [32, 36]. Currently, two species, namely E. bieneusi and E. hepatopenaei, are recognised [39, 40]. Enterocytozoon bieneusi is known to occur in eutherian mammals and in birds, and E. hepatopenaei has been recorded in crustaceans, such as the black tiger shrimp (Penaeus monodon) [40] and the whiteleg shrimp (Penaeus vannamei) [41]. However, due to limited SSU sequence similarity (85.2$85.9 \%)$ to E. bieneusi and a relatively close relationship with Enterospora nucleophila (cf. Fig. 2), there has been some reservation about the specific status of $E$. hepatopenaei (cf. [32]). With the present molecular findings, we now introduce added complexity by proposing that Enterocytozoon genotypes MWC_m2, MWC_m3 and MWC_m4 from the eastern grey kangaroo or swamp wallaby (cf. Figs. 1 and 2) represent a new species. Clearly, in the future, comprehensive phylogenetic analyses of nuclear genomic/ribosomal data sets (with suitable levels of phylogenetic signal) for a wide range of Enterocytozoon taxa should be undertaken to gain clarity as to the number of species within the genus. Provided sufficient amounts of genomic DNA of such taxa from broad host and distributional ranges can be acquired, a nuclear genomic sequencing-assembly effort, followed by phylogenetic analyses of tens, hundreds or thousands of homologous gene sequences (cf. [42]), could significantly assist toward solving the taxonomy/systematics of Enterocytozoon.

Interestingly, E. bieneusi genotypes from kangaroos from previous studies $[10,11]$ were assigned to (new) Groups 10 and 11. As these kangaroos had been kept in captivity in the Kangaroo Breeding Research Base, Jiangsu Province [11] and Zhengzhou Zoo, Henan Province [10] in China, it is unclear whether these E. bieneusi genotypes (CHK1, CHK2, CSK1 and CSK2) originated from Australia and were specifically affiliated to the hosts (indeterminate species) [10], or whether one or more of these genotypes might have been acquired from one or more other animal species in the same captive environment in China. Regardless, the distinctiveness of many Enterocytozoon genotypes from macropodid marsupials (Group 11, Fig. 1) and the AT-richness (88.6$89.4 \%$ ) of the ITS sequences of these genotypes, with respect to those representing other groups, raises questions about the stringency of host specificity of these genotypes, and their co-evolution with their marsupial hosts. On a local scale, within a water catchment context, we now have the opportunity of evaluating, using established methods, whether any of the new Enterocytozoon genotypes (MWC_m1 to MWC_m4) recorded in marsupials also occur in other Australian-native and feral animals, or whether they are specific to macropods. On a national level, it would be interesting to extend molecular studies to a broader range of wild marsupial hosts in different states of Australia to gain a better understanding of the evolution of Enterocytozoon. Given that marsupials in Australia dispersed from South America 54.6-83.6 mya [43, 44], it would be interesting to explore, in an international context, whether, for example, kangaroos and wallabies in Australia harbour the same or similar Enterocytozoon taxa as their marsupial relatives in South America or other continents in a Gondwana context [44].

\section{Conclusions}

Exploring the genetic composition of Enterocytozoon bieneusi in animals and humans is important for understanding transmission patterns of disease (enterocytozoonosis), and for its prevention and control. By conducting the present molecular-phylogenetic investigation of Enterocytozoon DNA in faecal deposits $(n=$ 1365) from marsupials (common wombats, eastern grey kangaroos and swamp wallabies) living in catchment areas that supply the city of Melbourne (Australia) with drinking water, we identified or defined two operational taxonomic units (OTUs) or genotypes (NCF2 and MWC_m1) with zoonotic potential and three divergent genotypes (MWC_m2 to MWC_m4). Further studies of Enterocytozoon spp. from macropods and other animals should assist in elucidating the taxonomy and epidemiology of these species in Australia and elsewhere, and in assessing the public health risk of enterocytozoonosis.

\section{Additional files}

Additional file 1: Table S1. The numbers of DNA samples from faecal deposits from common wombats (Vombatus ursinus), eastern grey kangaroos (Macropus giganteus) and swamp wallabies (Wallabia bicolor) collected from 11 of Melbourne's water catchment areas from 2009 to 2017 and tested using PCR-based sequencing of the internal transcribed spacer (ITS) of nuclear ribosomal DNA. Within parentheses are the numbers of samples that were test-positive for Enterocytozoon using this technique and the codes representing currently recognised (NCF2) or novel (MWC_m1 
to MWC m4) operational taxonomic units (OTUs)/genotypes (cf. Table 1 and Additional file 2: Table S2). (XLSX $45 \mathrm{~kb}$ )

Additional file 2: Table S2. GenBank accession numbers of all internal transcribed spacer (ITS) of nuclear ribosomal DNA sequences used for phylogenetic analysis (Fig. 1), and associated information. Included here are ITS sequences of (i) E. bieneusi genotypes representing currently recognised Groups (1 to 9) from the published literature; (ii) five operational taxonomic units (OTUs)/genotypes of Enterocytozoon identified/defined in the present study; and (iii) the outgroups CD8 (KJ668735) and PtEbIX (DQ85585). (DOCX $75 \mathrm{~kb}$ )

Additional file 3: Table S3. GenBank accession numbers of all small subunit of ribosomal DNA (SSU) sequences used for phylogenetic analysis (Fig. 2), and associated information. Included here are SSU sequences of (i) key taxa of the family Enterocytozoonidae, including representatives of E. bieneusi from the published literature; (ii) four operational taxonomic units (OTUs)/genotypes of Enterocytozoon identified/defined in the present study; and (iii) the outgroup Parahepatospora carcini. (DOCX $42 \mathrm{~kb}$ )

Additional file 4: Table S4. Pairwise comparisons of differences/variation among the small subunit of nuclear ribosomal DNA gene sequences (SSU) ( 609 bp consensus length) representing (i) four operational taxonomic units (OTUs)/genotypes of Enterocytozoon identified/defined in the present study (bold-type); (ii) eight species of of the family Enterocytozoonidae; (iii) and the outgroup Parahepatospora carcini used for phylogenetic analysis (cf. Fig. 2; Additional file 3: Table S3). (XLSX 54 kb)

\section{Abbreviations}

AIC: Akaike information criteria; BI: Bayesian inference; ITS: Internal transcribed spacer of nuclear ribosomal DNA; LSU: Large subunit of nuclear ribosomal DNA; MCMC: Monte Carlo Markov Chain; OTU: Operational taxonomic unit; pp: Posterior probability; SSU: Small subunit of nuclear ribosomal DNA

\section{Acknowledgements}

Melbourne Water staff are gratefully acknowledged.

\section{Funding}

Research funding from the Melbourne Water Corporation and Australian Research Council (grant number LP160101299) (RBG and AVK) is gratefully acknowledged. Yan Zhang was the recipient of scholarships from the Chinese Scholarship Council (CSC) and The University of Melbourne.

\section{Availability of data and materials}

Nucleotide sequences reported in this paper are available in the GenBank database under accession numbers MG976584-MG976586 (SSU) and MG976812MG976817 (partial SSU, complete ITS and partial LSU).

\section{Authors' contributions}

Collected samples: AVK and SRH. Analysis and interpretation: YZ, AVK, TW and RBG. Wrote the paper: YZ, AVK and RBG. Supervision of project: AVK, TW and RBG. Grant funding: RBG and AVK. All authors read and approved the final manuscript.

\section{Ethics approval and consent to participate}

Not applicable.

\section{Consent for publication}

Not applicable.

\section{Competing interests}

The authors declare that they have no competing interests.

\section{Publisher's Note}

Springer Nature remains neutral with regard to jurisdictional claims in published maps and institutional affiliations.

\section{Author details}

'Department of Veterinary Biosciences, Melbourne Veterinary School, Faculty of Veterinary and Agricultural Sciences, The University of Melbourne, Parkville, Victoria 3010, Australia. ${ }^{2}$ Melbourne Water, Docklands, Victoria 3001, Australia.
Received: 5 March 2018 Accepted: 14 June 2018

Published online: 28 June 2018

\section{References}

1. Santín M, Fayer R. Microsporidiosis: Enterocytozoon bieneusi in domesticated and wild animals. Res Vet Sci. 2011;90:363-71.

2. Keeling P. Five questions about Microsporidia. PLoS Pathog. 2009;5: e1000489.

3. James TY, Kauff F, Schoch CL, Matheny PB, Hofstetter V, Cox CJ, et al. Reconstructing the early evolution of fungi using a six-gene phylogeny. Nature. 2006:443:818-22

4. Molina JM, Sarfati C, Beauvais B, Lémann M, Lesourd A, Ferchal F, et al. Intestinal microsporidiosis in human immunodeficiency virus-infected patients with chronic unexplained diarrhea: prevalence and clinical and biologic features. J Infect Dis. 1993;167:217-21.

5. Weber R, Bryan RT, Schwartz DA, Owen RL. Human microsporidial infections. Clin Microbiol Rev. 1994;7:426-61.

6. Didier ES, Weiss LM. Microsporidiosis: current status. Curr Opin Infect Dis. 2006;19:485-92.

7. Kotler DP, Orenstein JM. Clinical syndromes associated with microsporidiosis. Adv Parasitol. 1998;40:321-49.

8. Sak $B$, Brady $D$, Pelikánová $M$, Květoňová $D$, Rost $M$, Kostka $M$, et al. Unapparent microsporidial infection among immunocompetent humans in the Czech Republic. J Clin Microbiol. 2011;49:1064-70.

9. Santín-Durán M. Enterocytozoon bieneusi. In: Xiao L, Ryan U, Feng Y, editors. Biology of Foodborne Parasites. Boca Raton: CRC Press; 2015. p. 149-74.

10. Li J, Qi M, Chang Y, Wang R, Li T, Dong H, et al. Molecular characterization of Cryptosporidium spp., Giardia duodenalis, and Enterocytozoon bieneusi in captive wildlife at Zhengzhou Zoo, China. J Eukaryot Microbiol. 2015;62: 833-9.

11. Zhong Z, Tian Y, Song Y, Deng L, Li J, Ren Z, et al. Molecular characterization and multi-locus genotypes of Enterocytozoon bieneusi from captive red kangaroos (Macropus rufus) in Jiangsu Province. China. PLoS One. 2017:12:e0183249.

12. Santín M, Fayer R. Enterocytozoon bieneusi genotype nomenclature based on the internal transcribed spacer sequence: a consensus. J Eukaryot Microbiol. 2009:56:34-8.

13. Anane S, Attouchi H. Microsporidiosis: epidemiology, clinical data and therapy. Gastroenterol Clin Biol. 2010;34:450-64.

14. Galván AL, Magnet A, Izquierdo F, Fenoy S, Rueda C, Fernandez Vadillo C, et al. Molecular characterization of human-pathogenic microsporidia and Cyclospora cayetanensis isolated from various water sources in Spain: a yearlong longitudinal study. Appl Environ Microbiol. 2013;79:449-59.

15. Decraene V, Lebbad M, Botero-Kleiven S, Gustavsson AM, Löfdahl M. First reported foodborne outbreak associated with microsporidia, Sweden, October 2009. Epidemiol Infect. 2012;140:519-27.

16. Koehler AV, Haydon SR, Jex AR, Gasser RB. Cryptosporidium and Giardia taxa in faecal samples from animals in catchments supplying the city of Melbourne with drinking water (2011 to 2015). Parasit Vectors. 2016:9:315.

17. Stark D, van Hal S, Barratt J, Ellis J, Marriott D, Harkness J. Limited genetic diversity among genotypes of Enterocytozoon bieneusi strains isolated from HIV-infected patients from Sydney, Australia. J Med Microbiol. 2009;58:355-7.

18. Field A, Hing M, Milliken S, Marriott D. Microsporidia in the small intestine of HIV-infected patients. A new diagnostic technique and a new species. Med J Aust. 1993;158:390-4.

19. Zhang Y, Koehler AV, Wang T, Haydon SR, Gasser RB. First detection and genetic characterisation of Enterocytozoon bieneusi in wild deer in Melbourne's water catchments in Australia. Parasit Vectors. 2018;11:2.

20. Nolan MJ, Jex AR, Koehler AV, Haydon SR, Stevens MA, Gasser RB. Molecular-based investigation of Cryptosporidium and Giardia from animals in water catchments in southeastern Australia. Water Res. 2013;47:1726-40.

21. Triggs BE. Tracks, Scats and Other Traces: A Field Guide to Australian Mammals. Melbourne: Oxford University Press; 1996.

22. Dalén L, Götherström A, Angerbjörn A. Identifying species from pieces of faeces. Conserv Genet. 2004:5:109-11.

23. Katzwinkel-Wladarsch S, Lieb M, Helse W, Löscher T, Rinder H. Direct amplification and species determination of microsporidian DNA from stool specimens. Trop Med Int Health. 1996;1:373-8.

24. Kearse M, Moir R, Wilson A, Stones-Havas S, Cheung M, Sturrock S, et al. Geneious Basic: an integrated and extendable desktop software platform for the organization and analysis of sequence data. Bioinformatics. 2012;28: 1647-9. 
25. Raynaud L, Delbac F, Broussolle V, Rabodonirina M, Girault V, Wallon M, et al. Identification of Encephalitozoon intestinalis in travelers with chronic diarrhea by specific PCR amplification. J Clin Microbiol. 1998;36:37-40.

26. da Silva AJ, Schwartz DA, Visvesvara GS, De Moura H, Slemenda SB, Pieniazek NJ. Sensitive PCR diagnosis of infections by Enterocytozoon bieneusi (microsporidia) using primers based on the region coding for smallsubunit rRNA. J Clin Microbiol. 1996;34:986-7.

27. Huelsenbeck JP, Ronquist F. MRBAYES: Bayesian inference of phylogenetic trees. Bioinformatics. 2001;17:754-5.

28. Darriba D, Taboada GL, Doallo R, Posada D. jModelTest 2: more models, new heuristics and parallel computing. Nat Methods. 2012:9:772.

29. Karim MR, Dong H, Li T, Yu F, Li D, Zhang L, et al. Predomination and new genotypes of Enterocytozoon bieneusi in captive nonhuman primates in zoos in China: high genetic diversity and zoonotic significance. PLoS One. 2015:10:e0117991.

30. Li W, Li Y, Song M, Lu Y, Yang J, Tao W, et al. Prevalence and genetic characteristics of Cryptosporidium, Enterocytozoon bieneusi and Giardia duodenalis in cats and dogs in Heilongjiang Province, China. Vet Parasitol. 2015:208:125-34.

31. Zhang XX, Cong W, Lou ZL, Ma JG, Zheng WB, Yao QX, et al. Prevalence, risk factors and multilocus genotyping of Enterocytozoon bieneusi in farmed foxes (Vulpes lagopus), northern China. Parasit Vectors. 2016;9:72.

32. Bojko J, Clark F, Bass D, Dunn AM, Stewart-Clark S, Stebbing PD, et al. Parahepatospora carcini n. gen., n. sp., a parasite of invasive Carcinus maenas with intermediate features of sporogony between the Enterocytozoon clade and other microsporidia. J Invertebr Pathol. 2017;143:124-34.

33. Vossbrinck CR, Debrunner-Vossbrinck BA. Molecular phylogeny of the Microsporidia: ecological, ultrastructural and taxonomic considerations. Folia Parasitol. 2005;52:131-42.

34. Zhu X, Wittner M, Tanowitz HB, Kotler D, Cali A, Weiss LM. Small subunit rRNA sequence of Enterocytozoon bieneusi and its potential diagnostic role with use of the polymerase chain reaction. J Infect Dis. 1993;168:1570-5.

35. Freeman MA, Kasper JM, Kristmundsson Á. Nucleospora cyclopteri n. sp., an intranuclear microsporidian infecting wild lumpfish, Cyclopterus lumpus L., in Icelandic waters. Parasit Vectors. 2013;6:49.

36. Docker MF, Kent ML, Hervio DM, Khattra JS, Weiss LM, Cali A, et al. Ribosomal DNA sequence of Nucleospora salmonis Hedrick, Groff and Baxa, 1991 (Microsporea: Enterocytozoonidae): implications for phylogeny and nomenclature. J Eukaryot Microbiol. 1997:44:55-60.

37. Diamant A, Rothman SB, Goren M, Galil BS, Yokes MB, Szitenberg A, et al. Biology of a new xenoma-forming gonadotropic microsporidian in the invasive blotchfin dragonet Callionymus filamentosus. Dis Aquat Organ. 2014;109:35-54.

38. McNeill J, Barrie F, Buck W, Demoulin V, Greuter W, Hawksworth D, et al. International code of nomenclature for algae, fungi and plants (Melbourne code). Regnum Vegetabile 154. Königstein: A.R.G. Gantner Verlag KG; 2012.

39. Desportes I, Le Charpentier Y, Galian A, Bernard F, Cochand-Priollet B, Lavergne A, et al. Occurrence of a new microsporidan-Enterocytozoon bieneusi n. g., n. sp., in the enterocytes of a human patient with AIDS. J Protozool. 1985:32:250-4.

40. Tourtip S, Wongtripop S, Stentiford GD, Bateman KS, Sriurairatana S, Chavadej J, et al. Enterocytozoon hepatopenaei sp. nov. (Microsporida: Enterocytozoonidae), a parasite of the black tiger shrimp Penaeus monodon (Decapoda: Penaeidae): fine structure and phylogenetic relationships. J Invertebr Pathol. 2009;102:21-9.

41. Rajendran K, Shivam S, Praveena PE, Rajan JJS, Kumar TS, Avunje S, et al. Emergence of Enterocytozoon hepatopenaei (EHP) in farmed Penaeus (Litopenaeus) vannamei in India. Aquaculture. 2016;454:272-80.

42. Korhonen PK, Pozio E, La Rosa G, Chang BC, Koehler AV, Hoberg EP, et al. Phylogenomic and biogeographic reconstruction of the Trichinella complex. Nat Commun. 2016;7:105-13.

43. Beck RMD. An 'ameridelphian'marsupial from the early eocene of Australia supports a complex model of southern hemisphere marsupial biogeography. Naturwissenschaften. 2012;99:715-29.

44. Beck RMD. The biogeographical history of non-marine mammaliaforms in the Sahul region. In: Ebach M, editor. Handbook of Australasian Biogeography. Boca Raton: CRC Press; 2017. p. 329-44.

\section{Ready to submit your research? Choose BMC and benefit from:}

- fast, convenient online submission

- thorough peer review by experienced researchers in your field

- rapid publication on acceptance

- support for research data, including large and complex data types

- gold Open Access which fosters wider collaboration and increased citations

- maximum visibility for your research: over $100 \mathrm{M}$ website views per year

At BMC, research is always in progress.

Learn more biomedcentral.com/submissions 